\title{
Hobnail hemangioma of the skin in a juvenile patient
}

\author{
Anna Ostrowska', Anna Golonka², Paweł Bojar', Iwona Paśnik', Justyna Szumiło' \\ ${ }^{1}$ Department of Clinical Pathomorphology, Medical University of Lublin, Poland \\ ${ }^{2}$ Department of Pediatric Surgery and Traumatology, Medical University of Lublin, Poland
}

Ostrowska A, Golonka A, Bojar P, Paśnik I, Szumiło J. Hobnail hemangioma of the skin in a juvenile patient. J Pre-Clin Clin Res. 2015; 9(1): 89-91. doi: 10.5604/18982395.1157585

\begin{abstract}
I Abstract
Hobnail hemangioma is a rare, benign, vascular lesion with peculiar but commonly misleading morphology, located mostly on the extremities or trunk, and affects young and middle-aged adults with a slightly male predominance. A new typical case in a 17-year-old girl is presented. A single, small, painless skin lesion appeared in an early childhood on the lateral part of the right thigh, and was linked with scratching a previous pigmented lesion. Microscopic examination of the completely removed lesion revealed typical morphology. Prominent endothelial cells were podoplanin-negative and CD34-positive. There was no recurrence after four years follow-up.
\end{abstract}

\section{Key words}

hobnail hemangioma, hobnail cells, vascular malformation, vascular neoplasms

\section{INTRODUCTION}

Hobnail hemangioma $(\mathrm{HH})$ is a rare, benign, vascular lesion with peculiar morphology. It was first described in 1988 by Santa Cruz and Aronberg [1] as a targetoid hemosiderotic hemangioma because of its characteristic 'targetoid' appearance, with a violaceus papule surrounded by an ecchymotic or brown ring that can expand and then disappear. However, in later descriptions, other morphological features were also presented [2]. HH affects mainly young and middle-aged adults with a slightly male predominance [3]. The most common localization is the skin of the extremities and trunk, but unusual sites like the oral and nasal mucosa have been also reported $[4,5]$. Trauma of the normal skin or pre-existing hemangioma is regarded as a predisposing factor, but sex hormones may also influence the morphology, resulting in the cyclic changes of diameter and peripheral colour [6]. It is suggested that $\mathrm{HHs}$ may result in connections and microshunts between lymphatic and adjacent blood vessels in the skin [7].

A new typical case of skin hobnail hemangioma in a juvenile patient is presented.

\section{CASE REPORT}

A 17-year-old female was admitted to the outpatient clinic with a single painless lesion, a $0.4 \mathrm{~cm}$ in diameter, ill-defined, brownish macula, located on the lateral part of the right thigh. The patient noticed slight enlargement of the lesion and change in colour from pale to almost dark black; however, none of these features depended on her menstrual cycle or others external factors. Bleeding or ulceration was not observed. The lesion had appeared in an early childhood, and was linked with scratching a pigmented lesion at the same locality.

Clinically, a provisional diagnosis of melanocytic nevus was made. The lesion was removed with wide margins.

Address for correspondence: Anna Ostrowska, Department of Clinical Patomorphology, Medical Univesity of Lublin, 7 Jaczewskiego Street, 20-090 Lublin, Poland E-mail: aniaoostrowska@gmail.com

Received: 18 August 2014; accepted: 20 November 2014
Microscopic examination revealed few irregular, dilated, thin-walled vascular channels located mostly in the papillary dermis [Fig. 1A] and lined with prominent hobnail, endothelial cells [Fig. 1B]. Endothelium was podoplanin
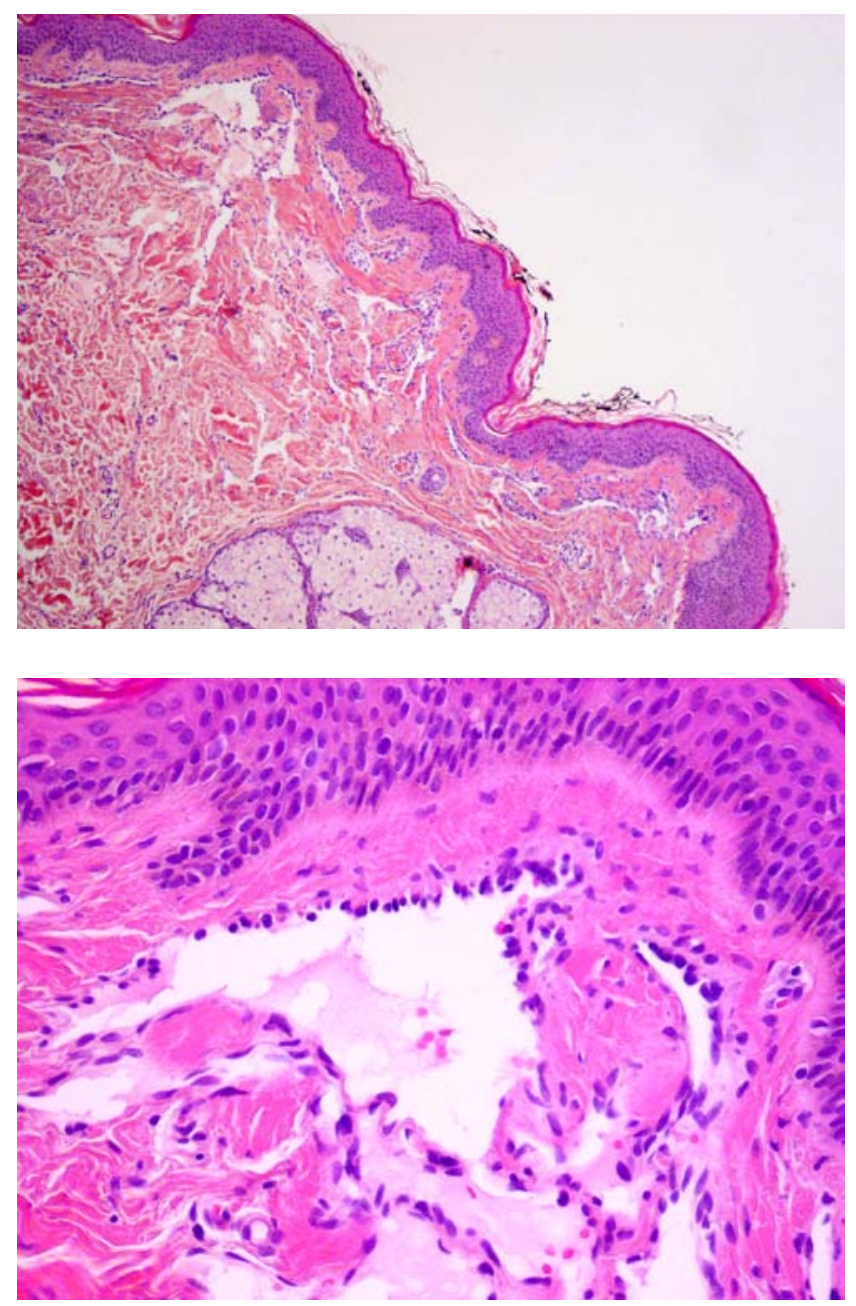

Figure 1. Irregular, dilated, thin-walled vascular channels located in the papillary dermis $(A)$, lined with prominent endothelial cells $(B)$ in hobnail hemangioma of the skin $(H+E$. Objective magnification $A-5 \times$; $B-20 X)$. 

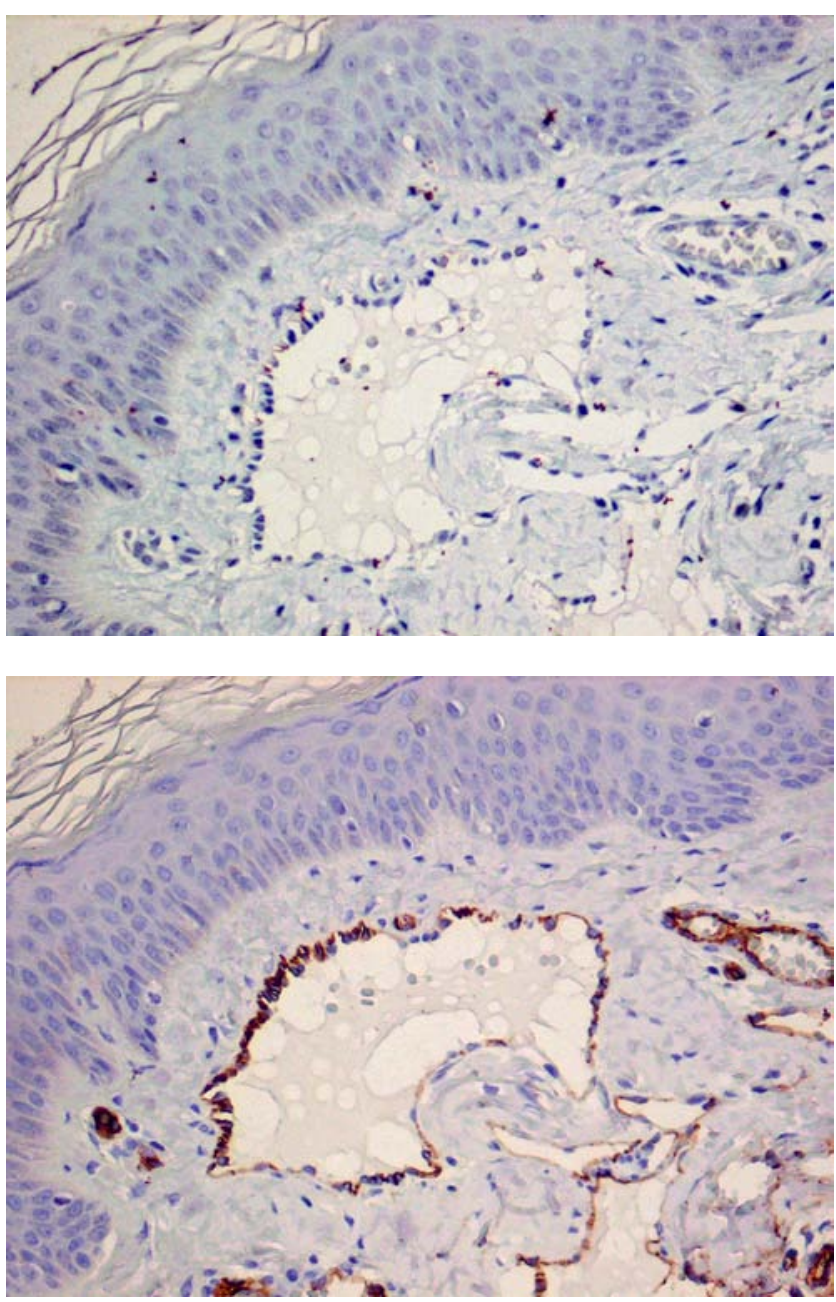

Figure 2. Negative immunoreaction with podoplanin (D2-40) (A) and positive with CD34 (B) in endothelial cells of hobnail hemangioma (Dako, EnVision+/HRP. Objective magnification $A, B-20 \times)$

(D2-40) negative [Fig. 2A], but CD34-positive [Fig. 2B]. Some scattered hemosiderin deposits were seen in the stroma. The resection margins were intact. Based on morphology of the lesion, hobnail hemangioma was diagnosed. There was no recurrence after four years follow-up.

\section{DISCUSSION}

Despite the first descriptions, hobnail hemangioma presents diversity in gross appearance and may cause many difficulties with making diagnosis. It usually occurs as a single solid lesion fluctuates with colour (blue-purple, yellow to green, violet, red, dark brown) and size $(2-15 \mathrm{~mm})$ [8]. Congenital and multiple HHs are uncommon [9]. In most cases, including the current one, it was completely asymptomatic, but sporadically painful lesions have been reported [8]. Unlike the presented patients changes in diameter and colour dependent on a menstrual cycle and pregnancy have been noted in a few cases, but estrogen, progesterone and androgen receptors were not revealed [10].

Histologically, HHs are characterized by a biphasic growth pattern of dilated vascular structures in the papillary dermis and narrow vascular spaces in the deeper part of the skin, and dissecting collagen fibres [4]. Vessels are lined by plump, exophytic endothelial cells showing a hobnail appearance. In some cases, the endothelial cells are hobnail-like in the superficial part of the lesion, but in the deeper part they are flat and inconspicuous [5]. Hemosiderin deposits, extravasated erythrocytes and mononuclear inflammatory infiltrate are also observed $[3,5,11]$. Due to their gross and microscopic features,

HHs have to be differentiated from variety of pathological lesions including other vascular tumors and malformations, especially cherry angioma, angiokeratoma, haemangioma in pregnancy, low grade angiosarcoma and Kaposi sarcoma, as well as melanocytic naevi, melanoma, dermatofibroma, seborrhoeic keratosis, and pigmented basal cell carcinoma. Some non-neoplastic lesions, e.g. insect bite reaction and endometriosis have also to be taken into consideration $[7,12]$. Careful clinical investigation together with dermoscopy and microscopic examination result in a correct final diagnosis [12]. Based on the above-mentioned data, the presented case is typical, and its appearance and development seems to be closely linked with mild local trauma.

The histogenesis of $\mathrm{HH}$ remains unclear. Based on immunohistochemical reaction with endothelial cells markers, some authors suggest a lymphatic origin of $\mathrm{HH}$ [2] and propose the term 'superficial lymphatic malformation' [3] or 'superficial hemosyderotic lymphovascular malformation' [8] as more appropriate for this entity. Unlike the present study, in many reports $\mathrm{HHs}$ were positive for both lymphatic (D2-40), and vascular (CD34) endothelial cell markers that may indicate biphasic nature of the lesions $[5,11]$. A neoplastic nature of $\mathrm{HH}$ is also doubtful. Trinidade et al. [3] regarded $\mathrm{HH}$ as a kind of lymphatic malformation rather than true neoplasm on the basis of negative immunohistochemical reaction with WT1.

\section{CONCLUSIONS}

1. The exact origin of hobnail hemangioma is still elusive and its morphological and clinical features might be misleading. Previous reports may suggest a biphasic character of this lesion, carrying both lymphatic and vascular features.

2. The presented entity required exact analysis and careful differential diagnosis.

\section{REFERENCES}

1. Santa Cruz DJ, Aronberg J Targetoid hemosyderotic hemangioma. J Am Acad Dermatol. 1988; 19: 550-558.

2. Mentzel T, Partanen TA, Kutzner H Hobnail hemangioma ("targetoid hemosiderotic hemangioma"): clinicopathologic and immunohistochemical analysis of 62 cases. J Cutan Pathol. 1999: 26: 279-286.

3. Trinidade F, Kutzner H, Tellechea O, Requena L, Colmenero I Hobnail hemangioma reclassified as superficial lymphatic malformation: a study of 52 cases. J Am Acad Dermatol. 2012; 66: 112-115.

4. Hiremath SK, Charantimath S, Byakodi S, Bijjal S, Byakodi R, Sapra G Oral hobnail hemangioma: a case report. Arch Iran Med. 2013;16: 428-430.

5. Hejnold M, Dyduch G, Mojsa I, Okoń K Hobnail hemangioma: a immunohistochemical study and literature review. Pol J Pathol. 2012; 3: $189-192$.

6. Christensen LJ, Stone MS Trauma- induced simulator of targetoid hemosiderotic hemangioma. Am J Dermatopahol. 2001; 23: 221-223. 
7. Ibrahim M, Shwayder T Hobnail hemangioma in a nine-year-old boy: A rare case presented with dermoscopy. Dermatol Online J. 2010; 16: 7 .

8. Joyce JC, Keith PJ, Szabo S, Holland KE Superficial hemosidrotic lymphovascular malformation (hobnail hemangioma): a report of six cases. Pediatr Dermatol. 2014; 31: 281-285.

9. Yoon SY, Kwon HH, Jeon HC, Lee JH, Cho S Congenital and multiple hobnail hemangiomas. Ann Dermatol. 2011; 23: 539-543.

10. Ortiz-Re JA, Gonzalez-Ruiz A, San Miguel P, Alvarez C, Iglesias B, Antón I Hobnail hemangioma associated with the menstrual cycle. JEADV. 2005; 19: 367-369.
11. Franke FE, Steger K, Marks A, Kutzner H, Mentzel T Hobnail hemangiomas (targetoid hemosiderotic hemangiomas) are true lymphangiomas. J Cutan Pathol. 2004; 31: 362-367.

12. Sahin MT, Demir MA, Gunduz K, Ozturkcan S, Türel-Ermertcan A Targetoid haemosiderotic haemangioma: dermoscopic monitoring of three cases and review of the literature. Clin Exp Dermatol. 2005; 30: $672-676$. 